\title{
Blowup for Nonlocal Nonlinear Diffusion Equations with Dirichlet Condition and a Source
}

\author{
Guosheng Zhang ${ }^{1,2}$ and Yifu Wang ${ }^{2}$ \\ ${ }^{1}$ School of Mathematical Sciences, Capital Normal University, Beijing 100048, China \\ ${ }^{2}$ School of Mathematical Sciences, Beijing Institute of Technology, Beijing 100081, China \\ Correspondence should be addressed to Guosheng Zhang; guosheng-zhang@163.com
}

Received 4 June 2013; Accepted 25 September 2013

Academic Editor: Julio Rossi

Copyright ( 2013 G. Zhang and Y. Wang. This is an open access article distributed under the Creative Commons Attribution License, which permits unrestricted use, distribution, and reproduction in any medium, provided the original work is properly cited.

This paper is concerned with a nonlocal nonlinear diffusion equation with Dirichlet boundary condition and a source $u_{t}(x, t)=$ $\int_{-\infty}^{+\infty} J((x-y) / u(y, t)) d y-u(x, t)+u^{p}(x, t), x \in(-L, L), t>0, u(x, t)=0, x \notin(-L, L), t \geq 0$, and $u(x, 0)=u_{0}(x) \geq 0, x \in(-L, L)$, which is analogous to the local porous medium equation. First, we prove the existence and uniqueness of the solution as well as the validity of a comparison principle. Next, we discuss the blowup phenomena of the solution to this problem. Finally, we discuss the blowup rates and sets of the solution.

\section{Introduction}

Since the long-range effects are taken into account, nonlocal diffusion equations of the form

$$
\begin{aligned}
\frac{\partial}{\partial t} u(x, t) & =J * u-u(x, t) \\
& =\int_{\mathbb{R}^{N}} J(x-y)(u(y, t)-u(x, t)) d y
\end{aligned}
$$

have been widely used to model the dispersal of a species (see [1-7] and references therein). In fact, as stated in [7], if $u(x, t)$ is thought of as the density of a species at the point $x$ at time $t$ and $J(x-y)$ is thought of as the probability distribution of jumping from location $y$ to location $x$, then $\int_{\mathbb{R}^{N}} J(x-$ $y) u(y, t) d y$ is the rate at which individuals are arriving to position $x$ from all other places and $-u(x, t)=-\int_{\mathbb{R}^{N}} J(x-$ $y) u(x, t) d y$ is the rate at which they are leaving location $x$ to travel to all other sites. It is known that (1) shares many properties with the classical heat equation $u_{t}=\Delta u$, such that bounded stationary solutions are constant, a maximum principle holds for both of them, and perturbations propagate with infinite speed (see [7]). However, there is no regularizing effect in general (see [8]).
Another classical equation that has been used to model diffusion is the well-known porous medium equation $u_{t}=$ $\Delta u^{m}$ with $m>1$. This equation also shares several properties with the heat equation, but there is a fundamental difference; in this case we have finite speed of propagation. Properties of solutions to the porous medium equation, particularly the blowup phenomena of the solution, have been largely studied over the past years. See, for example, [9-12] and references therein.

In $[13,14]$, a nonlocal model for diffusion that is analogous to the local porous medium equation is studied. In this model the probability distribution of jumping from location $y$ to location $x$ is given by $J((x-y) / u(y, t))(1 / u(y, t))$ when $u(y, t)>0$ and 0 otherwise. In this case the rate at which individuals are arriving to position $x$ from all other places is $\int_{\mathbb{R}} J((x-y) / u(y, t)) d y$, and the rate at which they are leaving location $x$ to travel to all other sites is $-u(x, t)=-\int_{\mathbb{R}} J((x-$ $y) / u(y, t)) d y$. As before this consideration, in the absence of external sources, leads immediately to the fact that the density $u(x, t)$ has to satisfy

$$
u_{t}(x, t)=\int_{\mathbb{R}} J\left(\frac{x-y}{u(y, t)}\right) d y-u(x, t) .
$$


In [15], Bogoya and Elorreaga studied the following nonlocal equation:

$$
\begin{gathered}
u_{t}(x, t)=\int_{\mathbb{R}^{N}}\left(J\left(\frac{x-y}{u^{\alpha}(y, t)}\right) u^{1-N \alpha}(y, t)\right. \\
\left.-J\left(\frac{x-y}{u^{\alpha}(x, t)}\right) u^{1-N \alpha}(x, t)\right) d y \\
+f(u(x, t)), \quad x \in \Omega, \\
u(x, t)=0, \quad x \notin \Omega, \\
u(x, 0)=u_{0}(x), \quad x \in \Omega .
\end{gathered}
$$

They proved the existence and uniqueness of the solution as well as the validity of a comparison principle and also discussed the blowup phenomena of the solution for some sources.

In the present paper, we are concerned with the following nonlocal "Dirichlet" boundary value problem with a source:

$$
\begin{aligned}
u_{t}(x, t)= & \int_{-\infty}^{+\infty} J\left(\frac{x-y}{u(y, t)}\right) d y \\
& -u(x, t)+u^{p}(x, t), \quad x \in(-L, L), t>0, \\
u & (x, t)=0, \quad x \notin(-L, L), t \geq 0, \\
u(x, 0)= & u_{0}(x)=c+w_{0}(x), \quad x \in(-L, L) .
\end{aligned}
$$

Here $p \geq 1$ and $c \geq 0$. Let $J: \mathbb{R} \rightarrow \mathbb{R}$ be a nonnegative, smooth function, with $\int_{\mathbb{R}} J(z) d z=1$, supported in $[-1,1]$, symmetric, and strictly decreasing in $[0,1]$. We assume that $w_{0} \in L^{1}(\mathbb{R})$ is a nonnegative function.

In this model, it is assumed that no individual can survive outside of the domain $(-L, L)$. Therefore, the density must be zero there. However, individuals are allowed to jump outside the domain (where they die instantaneously). This is what we call Dirichlet boundary conditions.

For the convenience of the statement of our results, denoting $\Omega=(-L, L)$, some related definitions are introduced in the following.

Definition 1. A nonnegative function $\bar{u} \in C\left([0, T) ; L^{1}\right)$ is a supersolution of the problem (4) if it satisfies

$$
\begin{aligned}
& \frac{\partial}{\partial t} \bar{u}(x, t) \geq \int_{-\infty}^{+\infty} J\left(\frac{x-y}{\bar{u}(y, t)}\right) d y \\
&-\bar{u}(x, t)+\bar{u}^{p}(x, t), \quad x \in(-L, L), t>0, \\
& \bar{u}(x, t)=0, \quad x \notin(-L, L), t \geq 0, \\
& \bar{u}(x, 0) \geq c+w_{0}(x), \quad x \in(-L, L) .
\end{aligned}
$$

The subsolution is defined similarly by reversing the inequalities. Furthermore, if $u$ is a supersolution as well as subsolution, then we call it a solution of the problem (4).
Definition 2. A solution $u(x, t)$ of the problem (4) is called a global solution if supremum norm $\|u(\cdot, t)\|_{L^{1}(\Omega)}$ is finite for all $t \geq 0$.

Definition 3. If there is a time $T_{\infty}<\infty$ such that a solution $u(x, t)$ of the problem (4) is bounded for all $T<T_{\infty}$ with $\lim _{t \rightarrow T_{\infty}}\|u(\cdot, t)\|_{L^{1}(\Omega)}=\infty$, then $u$ blows up in finite time $T_{\infty}$.

Now our main results can be stated as follows.

Theorem 4. If $p \geq 1, w_{0} \in L^{1}(\mathbb{R})$, and $c \geq 0$, then there exists a unique solution $u \in C\left([0, T) ; L^{1}(\mathbb{R})\right)$ to the problem (4).

Theorem 5 (blowup). Let $w_{0} \in C(\Omega)$ be nonnegative and nontrivial. If $p>1$, then the solution to the problem (4) blows up in finite time. If $p=1$, the solution to the problem (4) is global. Moreover, if $p>1$, one has the following estimate for the blowup time:

$$
T \leq \frac{1}{p-1}\left(\frac{|\Omega|}{\int_{\Omega} w_{0}(x) d x+c|\Omega|}\right)^{p-1} .
$$

The rest of the paper is organized as follows. In Section 2, we prove the existence and uniqueness of the solutions for the problem (4) and show a comparison principle for the solution. In Section 3, we deal with the blowup phenomenon for the problem (4) by the method of supersolutions and subsolutions. That is, the estimate of the blowup time, the blowup rates, and sets of the solution of the problem (4) are discussed.

\section{Existence and Uniqueness}

This section is devoted to the proof of the existence and uniqueness of the solution to the problem (4) via Banach's fixed point theorem. Simultaneously, the comparison principle for the solution of the problem (4) is also proved. To this end, it is convenient to give some preliminaries before giving its proof.

Fix $t_{0}>0$ and consider the Banach space $Y_{t_{0}}:=$ $C\left(\left[0, t_{0}\right] ; L^{1}(\mathbb{R})\right)$ with the norm

$$
|\|w\||_{Y_{t_{0}}}=\max _{0 \leq t \leq t_{0}}\|w(\cdot, t)\|_{L^{1}(\mathbb{R})} .
$$

We assume that $0 \leq w_{0}(x) \leq M$ a.e. in $\Omega$ and $A=2 M+1$. Let

$$
X_{t_{0}}=\left\{w \in C\left(\left[0, t_{0}\right] ; L^{1}(\mathbb{R})\right) \mid 0 \leq w \leq A\right\},
$$

which is a closed subset of $Y_{t_{0}}$. We will obtain the solution of the problem (4) in the form $u(x, t)=w(x, t)+c$, where $w$ is a fixed point of the operator $F_{w_{0}}: X_{t_{0}} \rightarrow X_{t_{0}}$ defined by

$$
\begin{gathered}
F_{w_{0}}(w)(x, t)=\int_{0}^{t} e^{-(t-s)}\left[\int_{\mathbb{R}} J\left(\frac{x-y}{w(y, s)+c}\right) d y\right. \\
\left.+(w(x, s)+c)^{p}\right] d s \\
+e^{-t} w_{0}-c\left(1-e^{-t}\right) .
\end{gathered}
$$


The following lemma is the main ingredient of the proof of Theorem 4.

Lemma 6. Let $w_{0}$ and $z_{0}$ be nonnegative functions such that $w_{0}, z_{0} \in L^{1}(\mathbb{R})$ and $w, z \in X_{t_{0}}$, and then

$$
\begin{aligned}
\|\| F_{w_{0}}(w)(x, t)-F_{z_{0}}(z)(x, t) \| \mid \\
\leq\left[1+p(A+c)^{p-1}\right]\left(1-e^{-t_{0}}\right)|\|w-z\|| \\
\quad+\left\|w_{0}-z_{0}\right\|_{L^{1}(\Omega)} .
\end{aligned}
$$

Therefore, if $t_{0}$ is small enough, $F_{w_{0}}$ is a strict contraction in $X_{t_{0}}$. Proof. From the definition of $F_{w_{0}}$, we have

$$
\begin{gathered}
\int_{\mathbb{R}}\left|F_{w_{0}}(w)(x, t)-F_{z_{0}}(z)(x, t)\right| d x \\
=\int_{\mathbb{R}} \mid \int_{0}^{t} e^{-(t-s)}\left[\int _ { \mathbb { R } } \left(J\left(\frac{x-y}{w(y, s)+c}\right)\right.\right. \\
\left.-J\left(\frac{x-y}{z(y, s)+c}\right)\right) d y \\
\left.+(w(x, s)+c)^{p}-(z(x, s)+c)^{p}\right] d s \\
+\int_{0}^{t} e^{-(t-s)} \int_{\mathbb{R} \mid} \mid \int_{\mathbb{R}}\left(J\left(\frac{x-y}{w(y, s)+c}\right)\right. \\
+e^{-t}\left(w_{0}-z_{0}\right)\left|d x e_{\mathbb{R}}\right| w_{0}-z_{0} \mid d x . \\
\left.+J\left(\frac{x-y}{z(y, s)+c}\right)\right) d y \mid d x d s \\
+\int_{\mathbb{R}}^{t}\left|(w(x, s)+c)^{p}-(z(x, s)+c)^{p}\right| d x d s
\end{gathered}
$$

Now set $A^{+}(s)=\{y \mid w(y, s) \geq z(y, s)\}$ and $A^{-}(s)=\{y \mid$ $w(y, s)<z(y, s)\}$. We have

$$
\begin{gathered}
\int_{\mathbb{R}}\left|\int_{\mathbb{R}}\left(J\left(\frac{x-y}{w(y, s)+c}\right)-J\left(\frac{x-y}{z(y, s)+c}\right)\right) d y\right| d x \\
\leq \int_{\mathbb{R}} \int_{A^{+}(s)}\left(J\left(\frac{x-y}{w(y, s)+c}\right)\right. \\
\left.-J\left(\frac{x-y}{z(y, s)+c}\right)\right) d y d x \\
+\int_{\mathbb{R}} \int_{A^{-}(s)}\left(J\left(\frac{x-y}{z(y, s)+c}\right)\right. \\
\left.-J\left(\frac{x-y}{w(y, s)+c}\right)\right) d y d x .
\end{gathered}
$$

Since the integrands are nonnegative, applying Fubini's theorem, we can get

$$
\begin{gathered}
\int_{\mathbb{R}} \int_{A^{+}(s)}\left(J\left(\frac{x-y}{w(y, s)+c}\right)-J\left(\frac{x-y}{z(y, s)+c}\right)\right) d y d x \\
=\int_{A^{+}(s)}(w(y, s)-z(y, s)) d y, \\
\int_{\mathbb{R}} \int_{A^{-}(s)}\left(J\left(\frac{x-y}{z(y, s)+c}\right)-J\left(\frac{x-y}{w(y, s)+c}\right)\right) d y d x \\
=\int_{A^{-}(s)}(z(y, s)-w(y, s)) d y .
\end{gathered}
$$

Therefore, we obtain

$$
\begin{gathered}
\int_{\mathbb{R}}\left|\int_{\mathbb{R}}\left(J\left(\frac{x-y}{w(y, s)+c}\right)-J\left(\frac{x-y}{z(y, s)+c}\right)\right) d y\right| d x \\
\quad \leq \int_{\mathbb{R}}|w(y, s)-z(y, s)| d y .
\end{gathered}
$$

Since $p \geq 1$ and $w, z \in X_{t_{0}}$, by the differential mid-value theorem, we deduce that

$$
\begin{aligned}
\int_{\mathbb{R}}\left|(w(x, s)+c)^{p}-(z(x, s)+c)^{p}\right| d x \\
\quad \leq p(A+c)^{p-1} \int_{\mathbb{R}}|w(x, s)-z(x, s)| d x .
\end{aligned}
$$

Furthermore, from the estimate (14) and (15), we get the desired estimate (10).

Next, we check that $F_{w_{0}}$ maps $X_{t_{0}}$ into $X_{t_{0}}$. Since $0 \leq w \leq$ $A$, for any $w \in X_{t_{0}}$, we have

$$
J\left(\frac{x-y}{A+c}\right) \geq J\left(\frac{x-y}{w(y, s)+c}\right) \geq J\left(\frac{x-y}{c}\right) .
$$

Hence, taking $t_{0} \leq \ln \left(\left(A+(A+c)^{p}\right) /\left(M+(A+c)^{p}\right)\right)$, we get that

$$
\begin{aligned}
F_{w_{0}}(w)(x, t) \geq & \int_{0}^{t} e^{-(t-s)} \int_{\mathbb{R}} J\left(\frac{x-y}{c}\right) d y d s \\
& +\int_{0}^{t} e^{-(t-s)}(w(x, s)+c)^{p} d s \\
& +e^{-t} w_{0}-c\left(1-e^{-t}\right) \\
= & c e^{-t}\left(e^{t}-e^{0}\right)+e^{-t} w_{0}(t)-c\left(1-e^{-t}\right) \\
& +\int_{0}^{t} e^{-(t-s)}(w(x, s)+c)^{p} d s \\
= & e^{-t} w_{0}(t)+\int_{0}^{t} e^{-(t-s)}(w(x, s)+c)^{p} d s \geq 0
\end{aligned}
$$




$$
\begin{aligned}
F_{w_{0}}(w)(x, t) \leq & \int_{0}^{t} e^{-(t-s)} \int_{\mathbb{R}} J\left(\frac{x-y}{A+c}\right) d y d s \\
& +\int_{0}^{t} e^{-(t-s)}(w(x, s)+c)^{p} d s \\
& +e^{-t} w_{0}-c\left(1-e^{-t}\right) \\
\leq & e^{-t}(A+c)\left(e^{t}-1\right)+e^{-t}(A+c)^{p}\left(e^{t}-1\right) \\
& +e^{-t} w_{0}-c\left(1-e^{-t}\right) \\
= & {\left[A+(A+c)^{p}\right]\left(1-e^{-t}\right)+e^{-t} w_{0} } \\
\leq & {\left[A+(A+c)^{p}\right]\left(1-e^{-t_{0}}\right)+w_{0} \leq A . }
\end{aligned}
$$

Thus, we conclude that $F_{w_{0}}(w)(x, t) \in X_{t_{0}}$.

Finally, choosing $t_{0} \leq \ln \left(\left(2 p(A+c)^{p-1}+2\right) /\left(2 p(A+c)^{p-1}+\right.\right.$ 1)), we have

$$
\begin{aligned}
\| F_{w_{0}} & (w)(x, t)-F_{w_{0}}(z)(x, t) \| \mid \\
& \leq\left[1+p(A+c)^{p-1}\right]\left(1-e^{-t_{0}}\right)|\|w-z\|| \\
\quad \leq & \frac{1}{2}|\|w-z\|| .
\end{aligned}
$$

Therefore, if $t_{0}$ is small enough, $F_{w_{0}}$ is a strict contraction in $X_{t_{0}}$. The proof is completed.

\subsection{The Proof of Theorem 4}

Proof. From Lemma 6, $F_{w_{0}}$ is a strict contraction in $X_{t_{0}}$ for $t_{0}$ small enough. By the Banach fixed point theorem, there exists only one fixed point of $F_{w_{0}}$ in $X_{t_{0}}$. This proves the existence and uniqueness of the solution of (4) in the time interval $\left[0, t_{0}\right]$. To continue, we may take $u\left(x, t_{0}\right)$ as initial data and obtain a unique solution of $(4)$ in the time interval $\left[0, t_{1}\right]$. If \|\|$w \|_{Y_{t_{1}}}<\infty$, arguing as before with $u\left(\cdot, t_{1}\right)$ as the initial datum, it is possible to extend the solution up to some interval $\left[0, t_{2}\right)$ for certain $t_{2}>t_{1}$. Hence, we can conclude that if the maximal time of the existence of the solution, $T$, is finite, then the solution blows up in $L^{1}(\Omega)$ norm; that is,

$$
\limsup _{t>T}\|u(x, t)\|_{L^{1}(\Omega)}=+\infty .
$$

Otherwise, the solution of the problem (4) is global.

Remark 7. From the proof of Theorem 4, the solution of (4) $u(x, t)$ is nonnegative.

Remark 8. If $w_{0} \in C(\Omega)$, then the solution of $(4) u(\cdot, t) \in$ $C(\Omega)$ for all $t \geq 0$.

To complete the proof of Theorem 5, we introduce the comparison principle for the problem (4) which is a very useful tool in studying diffusion problems.
Lemma 9. Let $\bar{u}$ and $\underline{u}$ be continuous supersolution and subsolution of the problem (4), respectively, and then $\underline{u}(x, t) \leq$ $\bar{u}(x, t)$ for all $(x, t) \in \Omega \times[0, T)$.

Proof. By an approximation procedure we restrict ourselves to consider strict inequalities for the supersolution. Indeed, we can take $\bar{u}(x, t)+\delta t+\delta(\delta>0)$ as a strict supersolution and take limit as $\delta \rightarrow 0$ at the end.

Hence, we consider strict inequalities for the supersolution and subsolution. Let $\bar{u}(x, 0)-\underline{u}(x, 0)>0$ for all $x \in \Omega$. Arguing by contradiction, we assume that there exist a first time $t_{0}>0$ and some point $x_{0} \in \Omega$ such that $\underline{u}\left(x_{0}, t_{0}\right)=$ $\bar{u}\left(x_{0}, t_{0}\right)$ and $\underline{u}(x, t) \leq \bar{u}(x, t)$ for all $(x, t) \in \Omega \times\left[0, t_{0}\right]$. Then, it holds that

$$
\begin{aligned}
0 & \leq \frac{\partial \underline{u}}{\partial t}\left(x_{0}, t_{0}\right)-\frac{\partial \bar{u}}{\partial t}\left(x_{0}, t_{0}\right) \\
& <\int_{\Omega}\left[J\left(\frac{x_{0}-y}{\underline{u}\left(y, t_{0}\right)}\right)-J\left(\frac{x_{0}-y}{\bar{u}\left(y, t_{0}\right)}\right)\right] d y \leq 0,
\end{aligned}
$$

and we reach the desired contradiction.

\section{Blowup Time, Blowup Rates, and Sets}

Once the existence and uniqueness of the solutions to the problem (4) are proved, we begin to analyze the blowup phenomenon for the problem (4).

\subsection{The Proof of Theorem 5}

Proof. For $p>1$, integrating in $x \in \mathbb{R}$ and $s \in(0, t)$ in (4), we get

$$
\begin{aligned}
\int_{-\infty}^{+\infty} u(x, t) d x= & \int_{-\infty}^{+\infty} u_{0}(x) d x \\
& +\int_{-\infty}^{+\infty} \int_{0}^{t} \int_{-\infty}^{+\infty} J\left(\frac{x-y}{u(y, s)}\right) d y d s d x \\
& +\int_{-\infty}^{+\infty} \int_{0}^{t}\left(u^{p}(x, s)-u(x, s)\right) d s d x
\end{aligned}
$$

Applying Fubini's theorem, we can obtain

$$
\begin{aligned}
\int_{\Omega} u(x, t) d x= & \int_{\Omega} u_{0}(x) d x \\
& +\int_{0}^{t} \int_{-\infty}^{+\infty} \int_{-\infty}^{+\infty} J\left(\frac{x-y}{u(y, s)}\right) d x d y d s \\
& +\int_{0}^{t} \int_{-\infty}^{+\infty}\left(u^{p}(x, s)-u(x, s)\right) d s d x
\end{aligned}
$$


Let $\tau=(x-y) / u(y, s)$; then $x=\tau u(y, s)+y$, and we have

$$
\begin{aligned}
\int_{\Omega} u(x, t) d x & \\
= & \int_{\Omega} u_{0}(x) d x+\int_{0}^{t} \int_{-\infty}^{+\infty} u(y, s) d y d s \\
& +\int_{0}^{t} \int_{-\infty}^{+\infty}\left(u^{p}(x, s)-u(x, s)\right) d s d x .
\end{aligned}
$$

That is,

$$
\int_{\Omega} u(x, t) d x=\int_{\Omega} u_{0}(x) d x+\int_{0}^{t} \int_{\Omega} u^{p}(x, s) d s d x
$$

Using Hölder inequality, we have

$$
\begin{aligned}
\frac{d}{d t} \int_{\Omega} u(x, t) d x & =\int_{\Omega} u^{p}(x, t) d x \\
& \geq|\Omega|^{1-p}\left(\int_{\Omega} u(x, t) d x\right)^{p} .
\end{aligned}
$$

Since $p>1$, we have that $\int_{\Omega} u(x, t) d x$ cannot be global; thus $u$ cannot be global either. Note that, by Theorem 4 , in this case, we have that $u(x, t)$ blows up in finite time in $L^{\infty}(\bar{\Omega})$ norm. Moreover, let $\int_{\Omega} u(x, t) d x=z(t)$, and we obtain

$$
\frac{d z}{d t} \geq|\Omega|^{1-p} z^{p}
$$

Integrating the above inequality, we have

$$
\frac{z^{1-p}(t)-z^{1-p}(0)}{1-p} \geq|\Omega|^{1-p} t
$$

Hence, it holds that

$$
\begin{aligned}
t & \leq \frac{|\Omega|^{p-1}\left(z^{1-p}(0)-z^{1-p}(t)\right)}{p-1} \\
& \leq \frac{|\Omega|^{p-1} z^{1-p}(0)}{p-1}=\frac{1}{p-1}\left(\frac{|\Omega|}{z(0)}\right)^{p-1},
\end{aligned}
$$

where $z(0)=\int_{\Omega} u(x, 0) d x=\int_{\Omega} w_{0}(x) d x+c|\Omega|$. Therefore, we can obtain the following estimate for the blowup time:

$$
T \leq \frac{1}{p-1}\left(\frac{|\Omega|}{\int_{\Omega} w_{0}(x) d x+c|\Omega|}\right)^{p-1} .
$$

For $p=1$, let us consider the ODE problem

$$
\begin{aligned}
& z^{\prime}(t)=z(t), \\
& z(0)=\max _{x \in \bar{\Omega}}\left\{w_{0}(x)+c, 1\right\} .
\end{aligned}
$$

Then, it follows that $z(t)=z(0) e^{t}$ and $z(t) \geq 1$ for $t>0$. We observe that $\int_{-\infty}^{\infty} J((x-y) / z(t)) d y=z(t)$. Therefore, $z(t)$ is a global supersolution of the problem (4). Thus, $u$ is global by comparison.
Concerning the blowup rate, that is, the speed at which solutions are blowing up, we find the following result.

Theorem 10 (blowup rates). Let $p>1$ and $u$ be a continuous solution to the problem (4) which blows up at time T. Then

$$
\lim _{t \rightarrow T^{-}}(T-t)^{1 /(p-1)} \max _{x \in \Omega} u(x, t)=\left(\frac{1}{p-1}\right)^{1 /(p-1)} .
$$

Proof. For every $t<T$, let $x_{0}(t) \in[-L, L]$ be such that $\max _{x \in \bar{\Omega}} u(\cdot, t)=u\left(x_{0}(t), t\right)$. Since $u(y, t) \leq u\left(x_{0}, t\right)$ for any $y \in \mathbb{R}$, we have

$$
J\left(\frac{x_{0}-y}{u(y, t)}\right) \leq J\left(\frac{x_{0}-y}{u\left(x_{0}, t\right)}\right)
$$

Changing the variable $\tau=\left(y-x_{0}\right) / u\left(x_{0}, t\right)$, then $y=x_{0}+u \tau$, $d y=u\left(x_{0}, t\right) d \tau$. Thus, we have

$$
\begin{aligned}
u_{t}\left(x_{0}, t\right) & =\int_{-\infty}^{+\infty} J\left(\frac{x_{0}-y}{u(y, t)}\right) d y+u^{p}\left(x_{0}, t\right)-u\left(x_{0}, t\right) \\
& =\int_{-\infty}^{+\infty} J(\tau) u\left(x_{0}, t\right) d \tau+u^{p}\left(x_{0}, t\right)-u\left(x_{0}, t\right) \\
& =u\left(x_{0}, t\right)+u^{p}\left(x_{0}, t\right)-u\left(x_{0}, t\right) \\
& =u^{p}\left(x_{0}, t\right) .
\end{aligned}
$$

Integrating the above inequality from $(t, T)$ and taking into account $p>1$, we obtain

$$
\max _{x \in \Omega} u(x, t) \geq(T-t)^{-1 /(p-1)}\left(\frac{1}{p-1}\right)^{1 /(p-1)} .
$$

To get the upper estimate, for any $(x, t) \in \bar{\Omega} \times[0, T)$, we observe that

$$
\begin{aligned}
u_{t}(x, t) & =\int_{-\infty}^{+\infty} J\left(\frac{x-y}{u(y, t)}\right) d y+u^{p}(x, t)-u(x, t) \\
& \geq-u(x, t)+u^{p}(x, t)=u^{p}(x, t)\left(1-u^{-(p-1)}(x, t)\right) .
\end{aligned}
$$

In particular

$$
\begin{aligned}
\max _{x \in \mathbb{R}} u_{t}(x, t) & \geq u_{t}(x, t) \\
& \geq \max _{x \in \mathbb{R}} u^{p}(x, t)\left(1-\max _{x \in \mathbb{R}} u^{-(p-1)}(x, t)\right) .
\end{aligned}
$$

Taking into account (34) in this expression, we get

$$
\max _{x \in \bar{\Omega}} u_{t}(x, t) \geq \max _{x \in \bar{\Omega}} u^{p}(x, t)(1-(p-1)(T-t)) .
$$

Integrating (37) in $(t, T)$, we obtain

$$
\max _{x \in \bar{\Omega}} u(x, t) \leq\left((p-1)(T-t)-\frac{1}{2}(p-1)^{2}(T-t)^{2}\right)^{-1 /(p-1)} .
$$

Taking the limit as $t \rightarrow T$, we will get the results. 
The blowup set, that is, the set of points at which the solutions blow up, is defined as follows:

$$
\begin{gathered}
B(u)=\{x \in \Omega \text {; there exists a finite time } T \\
\text { with } u(x, t) \longrightarrow \infty \text { as } t \nearrow T\} .
\end{gathered}
$$

Finally, we give the result concerning the blowup sets for the solution to the problem (4).

Theorem 11 (blowup sets). Let us consider the problem (4) with $p>2$. Given $x_{0} \in \Omega$ and $\varepsilon>0$, there exists an initial condition, $u_{0}$, such that the blowup set $B(u) \subset B_{\varepsilon}\left(x_{0}\right)=\{x \in$ $\left.\bar{\Omega} ;\left|x-x_{0}\right|<\varepsilon\right\}$.

Proof. Given $x_{0} \in \Omega$ and $\varepsilon>0$ we want to construct an initial condition $u_{0}$ such that

$$
B(u) \subset B_{\varepsilon}\left(x_{0}\right)=\left\{x \in \bar{\Omega} ;\left|x-x_{0}\right|<\varepsilon\right\} .
$$

To this end, we will consider $u_{0}$ concentrated near $x_{0}$ and small enough away from $x_{0}$.

Let $\varphi$ be a nonnegative smooth function such that

$$
\operatorname{supp}(\varphi) \subset B_{\varepsilon / 2}\left(x_{0}\right), \quad \varphi(x)>0 \quad \text { for } x \in B_{\varepsilon / 2}\left(x_{0}\right) \text {. }
$$

Now, let

$$
u_{0}(x)=M \varphi(x)+\delta
$$

We want to choose $M$ large and $\delta$ small in such a way that (40) holds.

First, note that, thanks to the estimate (6),

$$
\begin{aligned}
T & \leq \frac{1}{p-1}\left(\frac{|\Omega|}{\int_{\Omega}(M \varphi(x)+\delta) d x}\right)^{p-1} \\
& \leq \frac{1}{(p-1) M^{p-1}}\left(\frac{|\Omega|}{\int_{\Omega} \varphi(x) d x}\right)^{p-1},
\end{aligned}
$$

and taking $M$ large enough we can assume that $T$ is as small as we need. Now, using the upper bound for the blowup rate,

$$
\begin{aligned}
\max _{x \in \bar{\Omega}} u(x, t) & \leq\left((p-1)(T-t)-\frac{1}{2}(p-1)^{2}(T-t)^{2}\right)^{-1 /(p-1)} \\
& \leq C(T-t)^{-1 /(p-1)},
\end{aligned}
$$

we can obtain

$$
\begin{aligned}
u_{t}(\bar{x}, t) & =\int_{\mathbb{R}} J\left(\frac{\bar{x}-y}{u(y, t)}\right) d y-u(\bar{x}, t)+u^{p}(\bar{x}, t) \\
& \leq \int_{\mathbb{R}} J\left(\frac{\bar{x}-y}{u(y, t)}\right) d y+u^{p}(\bar{x}, t) \\
& \leq C(T-t)^{-1 /(p-1)}+u^{p}(\bar{x}, t)
\end{aligned}
$$

for any $\bar{x} \in \Omega$, where $T$ is small enough. Therefore, $u(\bar{x}, t)$ is a subsolution to

$$
w_{t}(t)=C(T-t)^{-1 /(p-1)}+w^{p}(\bar{x}, t) .
$$

And hence, if $u(\bar{x}, 0) \leq w(0)$, we have

$$
u(\bar{x}, t) \leq w(t)
$$

Now we just have to prove that a solution $w$ to (46) beginning with $w(0)=\delta$ remains bounded up to $t=T$, provided that $\delta$ and $T$ are small enough. To see this we use

$$
z(s)=(T-t)^{1 /(p-1)} w(t), \quad s=-\ln (T-t) .
$$

So we have

$$
\begin{aligned}
\frac{d z}{d s}=[ & -\frac{1}{p-1}(T-t)^{(1 /(p-1))-1} w \\
& \left.+(T-t)^{1 /(p-1)}\left(C(T-t)^{-(1 /(p-1))}+w^{p}\right)\right] e^{-s} \\
= & -\frac{1}{p-1}(T-t)^{1 /(p-1)} w \frac{e^{-s}}{T-t}+c e^{-s} \\
& +(T-t)^{-(1 /(p-1))} e^{-s} w^{p} \\
= & C e^{-s}+z^{p}(s)-\frac{1}{p-1} z(s), \quad z(-\ln T)=T^{(1 /(p-1))} \delta .
\end{aligned}
$$

Note that for $T$ and $\delta$ small it holds that $z^{\prime}(-\ln T)<0$. Indeed, we need

$$
C T-\frac{1}{p-1} \delta T^{1 /(p-1)}+\delta^{p} T^{p /(p-1)}<0 .
$$

For $p>2$, we have $(2-p) /(p-1)<0,1 /(p-1)>0$. If we choose the initial of the solution large enough to make sure $M$ large enough, then $T$ is small enough. So, for any fixed small $\delta>0$, it holds that $C<(1 /(p-1)) \delta T^{2-p} p-1-\delta^{p} T^{a /(p-1)}$. So the (50) holds. From this, it is easy to prove that $z^{\prime}(s)<0$ for all $s>-\ln T$. Therefore, $z(s) \rightarrow 0$ as $s \rightarrow \infty$. Going back to the equation verified by $z$, for any $\varepsilon>0$, we have

$$
\left(e^{(1 /(p-1)) s} z(s)\right)_{s} \leq C e^{-s}-\left(\frac{1}{p-1}-\varepsilon\right) z(s)
$$

Integrating the above inequality from $\left(s_{0}, s\right)$ and using that $p>2$, we have

$$
z(s) \leq C e^{(1 /(p-1)) s}
$$

In terms of $w(t)$ this bound implies that $w(t) \leq C$, for $0 \leq$ $t<T$. From the boundedness of $w$ and (47), we get $u(\bar{x}, t) \leq$ $w(t) \leq C$ for every $\bar{x} \in \bar{\Omega}-B_{\varepsilon}\left(x_{0}\right)$, as we desired. 


\section{References}

[1] P. W. Bates and A. Chmaj, "An integrodifferential model for phase transitions: stationary solutions in higher space dimensions," Journal of Statistical Physics, vol. 95, no. 5-6, pp. 1119-1139, 1999.

[2] P. W. Bates, P. C. Fife, X. Ren, and X. Wang, "Traveling waves in a convolution model for phase transitions," Archive for Rational Mechanics and Analysis, vol. 138, no. 2, pp. 105-136, 1997.

[3] C. Carrillo and P. Fife, "Spatial effects in discrete generation population models," Journal of Mathematical Biology, vol. 50, no. 2, pp. 161-188, 2005.

[4] X. Chen, "Existence, uniqueness, and asymptotic stability of traveling waves in nonlocal evolution equations," Advances in Differential Equations, vol. 2, no. 1, pp. 125-160, 1997.

[5] J. Coville, J. Dávila, and S. Martínez, "Nonlocal anisotropic dispersal with monostable nonlinearity," Journal of Differential Equations, vol. 244, no. 12, pp. 3080-3118, 2008.

[6] C. Cortázar, M. Elgueta, and J. D. Rossi, "Nonlocal diffusion problems that approximate the heat equation with Dirichlet boundary conditions," Israel Journal of Mathematics, vol. 170, pp. 53-60, 2009.

[7] P. Fife, "Some nonclassical trends in parabolic and paraboliclike evolutions," in Trends in Nonlinear Analysis, pp. 153-191, Springer, Berlin, Germany, 2003.

[8] E. Chasseigne, M. Chaves, and J. D. Rossi, "Asymptotic behavior for nonlocal diffusion equations," Journal de Mathématiques Pures et Appliquées, vol. 86, no. 3, pp. 271-291, 2006.

[9] J. L. Vázquez, The Porous Medium Equation, Oxford University Press, New York, NY, USA, 2007.

[10] V. A. Galaktionov, S. P. Kurdyumov, and A. A. Samarskil̆, "A parabolic system of quasi-linear equations I," Differential Equations, vol. 19, pp. 1558-1571, 1983.

[11] V. A. Galaktionov, S. P. Kurdyumov, and A. A. Samarskií, "A parabolic system of quasilinear equations II," Differential Equations, vol. 21, pp. 1049-1062, 1985.

[12] F. C. Li and C. H. Xie, "Global existence and blow-up for a nonlinear porous medium equation," Applied Mathematics Letters, vol. 16, no. 2, pp. 185-192, 2003.

[13] C. Cortazar, M. Elgueta, and J. D. Rossi, "A nonlocal diffusion equation whose solutions develop a free boundary," Annales Henri Poincaré, vol. 6, no. 2, pp. 269-281, 2005.

[14] M. Bogoya, R. Ferreira, and J. D. Rossi, "Neumann boundary conditions for a nonlocal nonlinear diffusion operator. Continuous and discrete models," Proceedings of the American Mathematical Society, vol. 135, no. 12, pp. 3837-3846, 2007.

[15] M. Bogoya and L. M. Elorreaga, "Problema de Dirichlet para una ecuación de difusión no local con fuente," Boletín de Matemáticas, vol. 19, no. 1, pp. 55-64, 2012. 


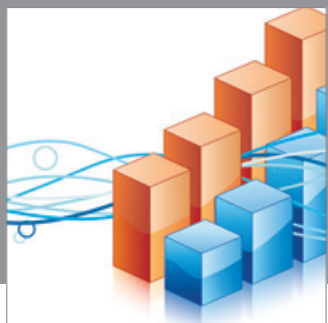

Advances in

Operations Research

mansans

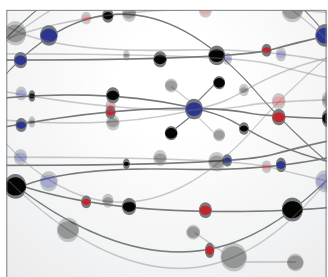

The Scientific World Journal
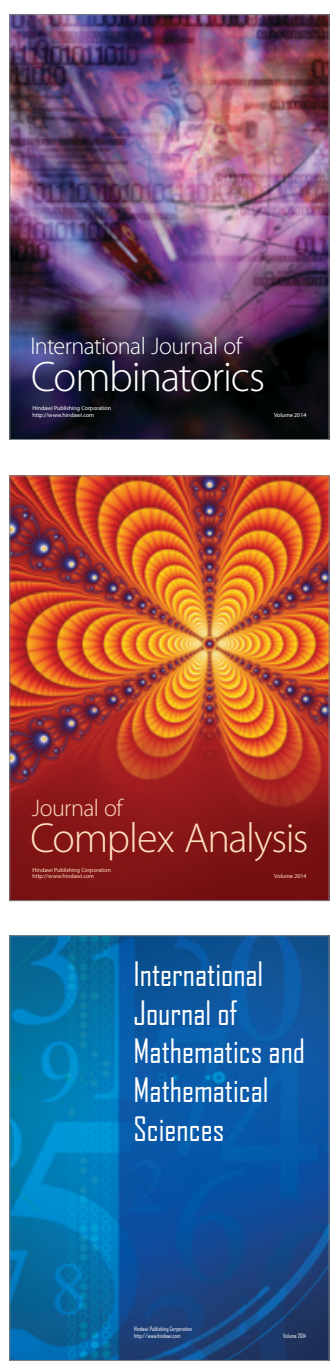
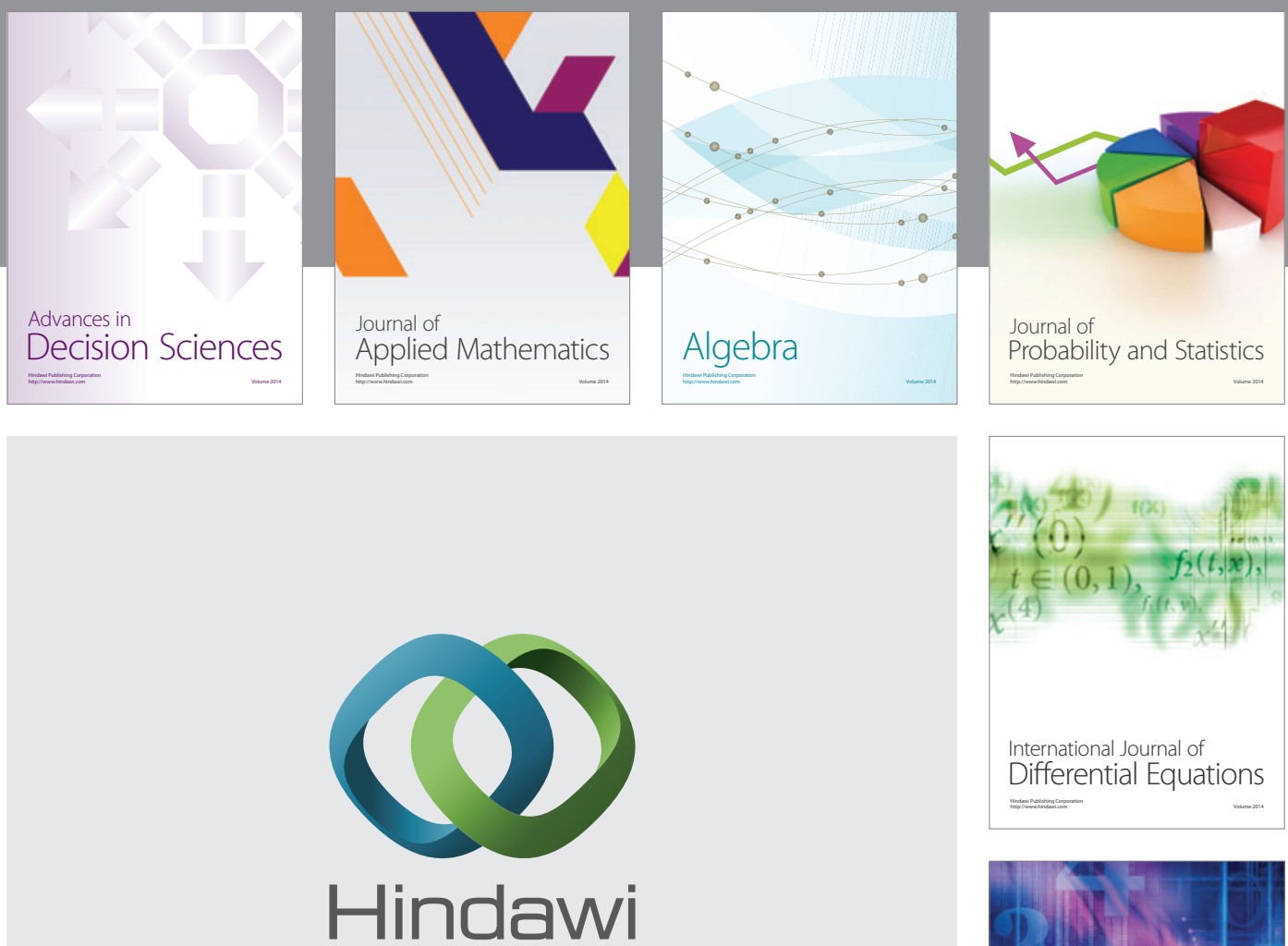

Submit your manuscripts at http://www.hindawi.com
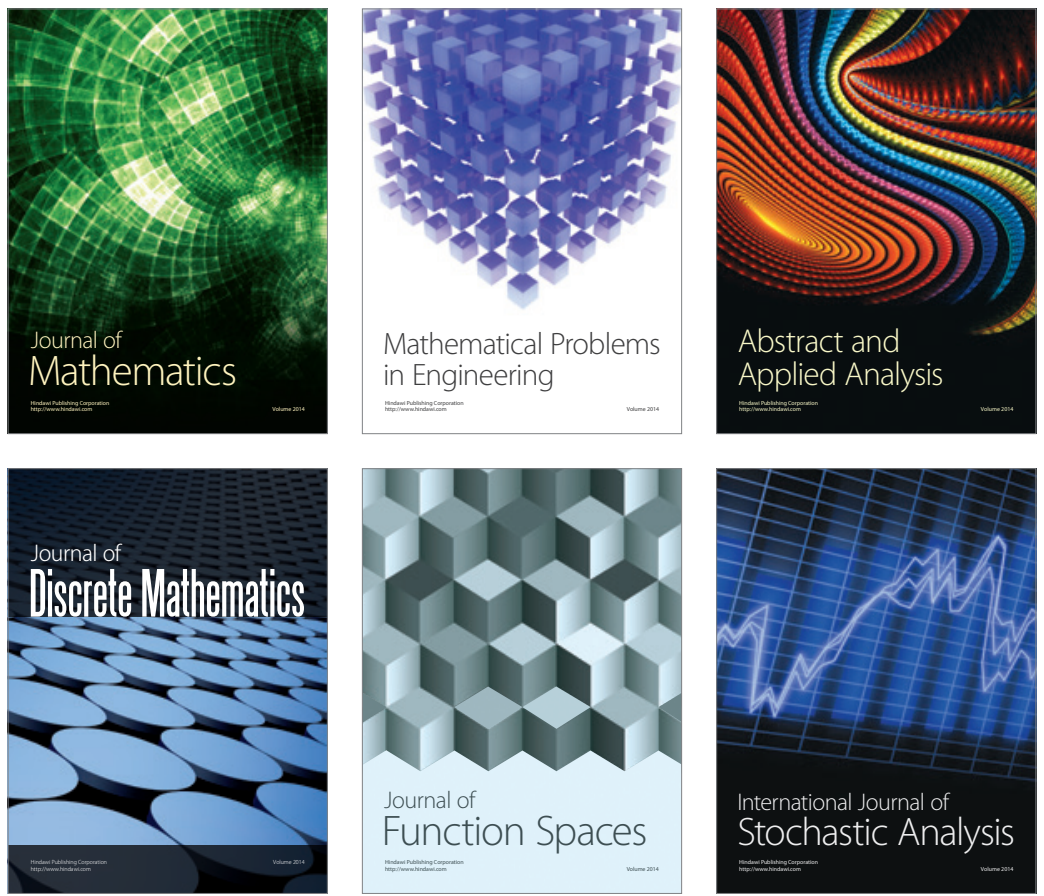

Journal of

Function Spaces

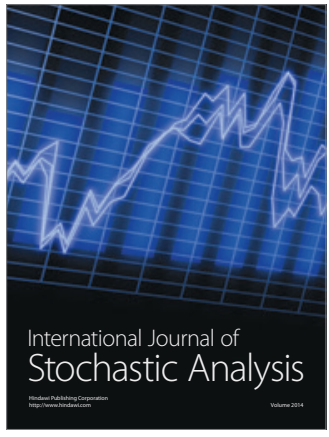

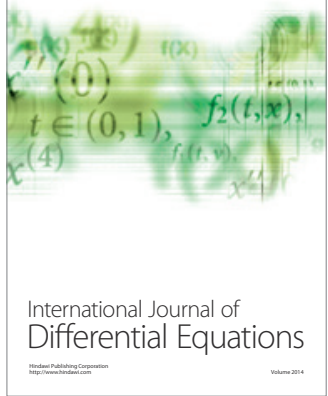
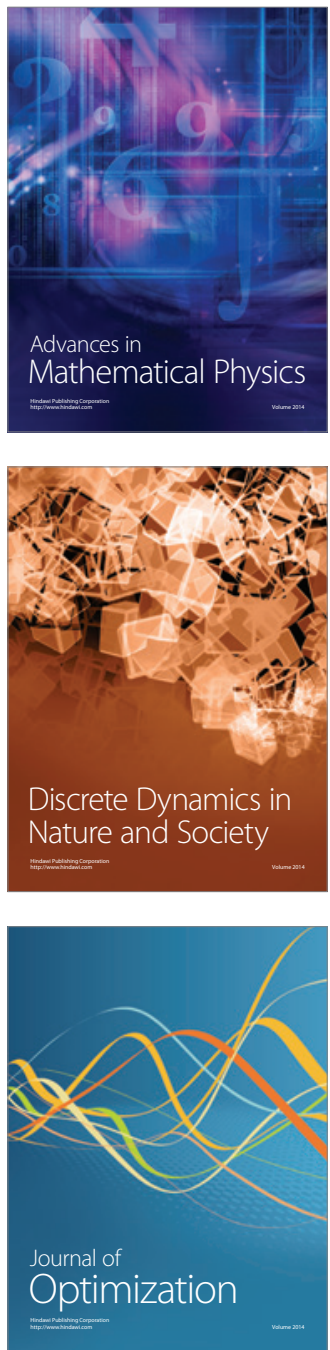